Pacific

Journal of

Mathematics

ON A NECESSARY CONDITION FOR SPANNERS IN A WEDGE

SUNG-HO PARK

Volume 234 No. 1

January 2008 


\title{
ON A NECESSARY CONDITION FOR SPANNERS IN A WEDGE
}

\author{
SUNG-HO PARK
}

\begin{abstract}
A spanner in a wedge or in a triangular pyramid is a compact embedded surface of constant mean curvature that does not meet the edge of the wedge or the triangular pyramid and meets the planes at constant angles. We show that the area of the planar region bounded by the boundary curve(s) on each plane, which is called the wetted region, of a spanner should be bigger than or equal to the area of the wetted region of the unique spherical spanner.
\end{abstract}

\section{Introduction}

A spanner $\Sigma$ in a wedge of $\mathbb{R}^{3}$ is a compact embedded surface of constant mean curvature $(\mathrm{cmc})$ that meets each plane of the wedge in constant angle and does not meet the edge of the wedge. Under these conditions, the boundary of $\Sigma$ determines a number of bounded domains $\Omega_{i}$ in the planes of the wedge; we refer to these domains as the wetted regions and require that the spanner $\Sigma$ along with the $\Omega_{i}$ bound an open volume in $\mathbb{R}^{3}$. In other words, $\Sigma$ is a compact capillary surface in a wedge in the absence of gravity which stays away from the edge. This means that a spanner represents an equilibrium configuration of some liquid drop inside the wedge, that is, not touching the edge, with respect to the energy

$$
E(\Sigma)=\sigma|\Sigma|-a_{1} \sigma\left|\Omega_{1}\right|-a_{2} \sigma\left|\Omega_{2}\right|
$$

Here $\sigma$ is the surface tension of the liquid-air interface, the $a_{i}$ are the adhesion constants between the fluid and the planes, and each $\Omega_{i}$ is the whole wetted region on each plane. We assume that the spanners are $C^{1}$ up to the boundary.

Let $X$ be the position vector for points on $\Sigma$, and let $\Delta$ be the intrinsic Laplacian on $\Sigma$. From the equation $\Delta X=2 H \vec{N}$ on $\Sigma(H$ is the mean curvature of $\Sigma$ ) and using the divergence theorem, we have

$$
\int_{\Sigma} 2 H \vec{N} d S=\oint_{\partial \Sigma} \vec{n} d s
$$

MSC2000: 53A05, 53A10.

Keywords: capillary surfaces, balancing formula, spanner. 
Here $\vec{N}$ is the outward unit normal of $\Sigma$, and $\vec{n}$ is the outward unit conormal of $\Sigma$ along $\partial \Sigma$. Since the mean curvature $H$ and the contact angles $\gamma_{1}$ and $\gamma_{2}$ are constant, we have (see for example [Concus et al. 2001])

$$
\sin \gamma_{1}\left|\partial \Omega_{1}\right|+2 H\left|\Omega_{1}\right|=0 \quad \text { and } \quad \sin \gamma_{2}\left|\partial \Omega_{2}\right|+2 H\left|\Omega_{2}\right|=0 .
$$

We note that the above formulas, which were proved for ring type spanners in [Concus et al. 2001] and are called the balancing formulas, are true for all spanners of any topological type (see the proof of balancing formulas for spanners in triangular pyramids in Section 2). In particular, they are still true when the wetted region on a plane is not connected. An immediate consequence of the balancing formulas is that the mean curvature vector of a spanner should point the inside of the liquid drop.

For ring type spanners in a wedge, McCuan [1997] used a spherical reflection technique to obtain a necessary condition ([Lemma 9] there or Lemma 1 in Section 2). Later, it was proved in [Park 2005], based on [McCuan 1997, Lemma 9], that every ring type spanner in a wedge is in fact spherical; such spanners are called spherical spanners.

In this paper, we obtain a necessary condition for spanners in a wedge. As an application, we give a simple proof for [McCuan 1997, Lemma 9]. The argument can be easily generalized to spanners in an octant or in a triangular pyramid. It also generalizes to higher dimensional spanners in wedges or in unbalanced polyhedral cones in higher dimensional Euclidean space $\mathbb{E}^{n+1}$ which are bounded by closed (n-1)-dimensional submanifold(s) on each face of the cone. We say that a polyhedral cone in Euclidean space is unbalanced if the unit normal vectors to each face of the polyhedral cone are linearly independent. We use the balancing formula for constant mean curvature surfaces and the isoperimetric inequality for plane domains. Our necessary condition says that the area of wetted region of spanners in a wedge or in an unbalanced polyhedral cones should be bigger than or equal to the area of wetted region of the unique spherical spanner.

I express my thanks to the referee for kind comments.

\section{Main result}

In the following, the unit normal vector field $N$ points to the outside of the spanner $\Sigma$, and the second fundamental form and the mean curvature $H$ are computed with respect to $N$. The surfaces are supposed to be $C^{1}$ up to the boundary.

Theorem 1. Let $\Sigma$ be a compact embedded cmc surface in a wedge. Suppose that $\Sigma$ does not meet the edge of the wedge and that the contact angle between $\Sigma$ and each face of the wedge is constant. Let $\gamma_{1}$ and $\gamma_{2}$ be the contact angles, and let $\Omega_{1}$ and $\Omega_{2}$ be the planar domains bounded by the boundary curves (the wetted 
regions). Then we have

$$
\left|\Omega_{i}\right| \geq \frac{\sin ^{2} \gamma_{i}}{H^{2}} \pi \quad \text { for } i=1,2 .
$$

Therefore the area of the wetted region of any spanner in a wedge is bigger than or equal to the area of the wetted region of the unique spherical spanner.

Proof. The isoperimetric inequality for domains in Euclidean plane says that

$$
\left|\partial \Omega_{i}\right|^{2} \geq 4 \pi\left|\Omega_{i}\right|
$$

Plugging (1) into this isoperimetric inequality, we have

$$
\left|\Omega_{i}\right| \geq \frac{\sin ^{2} \gamma_{i}}{H^{2}} \pi
$$

We note that $\sin \gamma_{i} /|H|$ is the radius of the wetted disk of the spherical spanner with mean curvature $H$ and contact angles $\gamma_{1}$ and $\gamma_{2}$.

Now let $\Sigma$ be a ring type spanner with contact angles $\gamma_{1}$ and $\gamma_{2}$. There is a conformal curvature coordinate $z=x+i y$ of $\Sigma$ such that the second fundamental form

$$
I I=h_{11}(d x)^{2}+2 h_{12} d x d y+h_{22}(d y)^{2}
$$

satisfies $h_{12} \equiv 0$ and $h_{11}-h_{22} \equiv$ constant [McCuan 1997]. Let $d s^{2}=\lambda|d z|^{2}$ be the induced metric. Then the mean curvature is

$$
H=\frac{1}{2 \lambda}\left(h_{11}+h_{22}\right) \text {. }
$$

Because $h_{11}-h_{22}=: c$ is constant, the principal curvatures are given by

$$
\kappa_{1}=H+\frac{c}{2 \lambda} \quad \text { and } \quad \kappa_{2}=H-\frac{c}{2 \lambda} .
$$

We note that $\kappa_{1}$ is the principal curvature in the radial direction and $\kappa_{2}$ is the principal curvature in the angular direction. We give a simple proof of [McCuan 1997, Lemma 9].

Lemma 1. Let $\Sigma$ be a ring type spanner in a wedge. Then the constant $c=h_{11}-h_{22}$ is nonpositive.

Proof. We note that the curvature $\kappa$ of $\partial \Omega_{1}$ is $\kappa_{2} / \sin \gamma_{1}=(H-c / 2 \lambda) / \sin \gamma_{1}$. If $c>0$, then $\partial \Omega_{1}$ can be placed inside the circle of radius $\sin \gamma_{1} /|H|$, and the inequality (3) cannot be satisfied. Hence, we must have $c \leq 0$.

Now we suppose that $\Sigma$ is a spanner in a triangular pyramid. For completeness, we include the proof of balancing formulas for spanners in triangular pyramids; see [Concus et al. 2001]. For $i=1,2,3$, let $\gamma_{i}$ be the contact angles, let $\Omega_{i}$ be the wetted regions, and let $N_{i}$ be the unit vectors perpendicular to the faces of 
the pyramid that are compatible with the surface normal $N$ of $\Sigma$. By integrating $\Delta X=2 H \vec{N}$ over $\Sigma$ and using the divergence theorem, we have

$$
\int_{\Sigma} 2 H \vec{N} d S=\int_{\Sigma} \Delta X d S=\int_{\partial \Sigma} \vec{n} d s=\sum_{i=1}^{3} \int_{\partial \Omega_{i}} \vec{n} d s .
$$

It is clear that

$$
\int_{\partial \Omega_{i}} \vec{n} d s=\int_{\partial \Omega_{i}}\left(\cos \gamma_{i} v_{i}+\sin \gamma_{i} N_{i}\right) d s,
$$

where $v_{i}$ is the outward conormal of $\Omega_{i}$ along $\partial \Omega_{i}$. Since $\gamma_{i}$ is constant,

$$
\int_{\partial \Omega_{i}} \sin \gamma_{i} N_{i} d s=\sin \gamma_{i}\left|\partial \Omega_{i}\right| N_{i}
$$

From the divergence theorem, we have $\int_{\partial \Omega_{i}^{j}} \cos \gamma_{i} v_{i} d s=0$ for each connected component $\partial \Omega_{i}^{j}$ of $\partial \Omega_{i}$, and

$$
\int_{\Sigma} \vec{N} d S+\sum_{i=1}^{3} \int_{\Omega_{i}} N_{i} d S=0
$$

Therefore we have

$$
\sum_{i=1}^{3} \int_{\Omega_{i}} 2 H N_{i} d S+\sum_{i=1}^{3} \sin \gamma_{i}\left|\partial \Omega_{i}\right| N_{i}=\sum_{i=1}^{3} 2 H\left|\Omega_{i}\right| N_{i}+\sum_{i=1}^{3} \sin \gamma_{i}\left|\partial \Omega_{i}\right| N_{i}=0 .
$$

Since $N_{1}, N_{2}$, and $N_{3}$ are linearly independent (that is, a triangular pyramid is unbalanced), we obtain the balancing formulas for spanners in triangular pyramids:

$$
\sin \gamma_{i}\left|\partial \Omega_{i}\right|+2 H\left|\Omega_{i}\right|=0 \quad \text { for } i=1,2,3 .
$$

The same argument can be applied to $\Delta X=n H \vec{N}$ to produce the balancing formulas for $n$-dimensional spanners in unbalanced polyhedral cones in the Euclidean space $\mathbb{E}^{n+1}$ :

$$
\sin \gamma_{i}\left|\partial \Omega_{i}\right|+n H\left|\Omega_{i}\right|=0 .
$$

Since the balancing formulas hold for spanners in triangular pyramids, we have

Theorem 2. Let $\Sigma$ be a compact embedded cmc surface in a triangular pyramid. Suppose that $\Sigma$ does not meet the edge and the vertex of the pyramid, and suppose that the contact angles between $\Sigma$ and the faces of the pyramid are constant on each face of the pyramid. For $i=1,2,3$, let $\gamma_{i}$ be the contact angles, and let $\Omega_{i}$ be the planar domains bounded by the boundary curves on each face (the wetted regions). Then

$$
\left|\Omega_{i}\right| \geq \frac{\sin ^{2} \gamma_{i}}{H^{2}} \pi \quad \text { for } i=1,2,3
$$


From the balancing formulas for higher dimensional spanners together with the isoperimetric inequality for domains in $\mathbb{E}^{n}$, we have

Theorem 3. Let $M$ be a compact embedded cmc hypersurface in an unbalanced polyhedral cone in $\mathbb{E}^{n+1}$ bounded by $(n-1)$-dimensional submanifold( $\left.s\right)$ on each face of the cone. Suppose that $M$ does not meet the edge and the vertex of the cone, and suppose that the contact angles between $M$ and the faces of the cone are constant on each face of the cone. Let $\gamma_{i}$ be the contact angles and $\Omega_{i}$ be the domains on each face of the cone bounded by the boundary submanifold(s) on each face (the wetted regions). Then we have

$$
\left|\Omega_{i}\right| \geq\left(\frac{\sin \gamma_{i}}{|H|}\right)^{n} \omega(n)
$$

where $\omega(n)$ is the volume of the unit ball in $\mathbb{E}^{n}$.

In unbalanced polyhedral cones, there exists a unique spherical spanner for given mean curvature $H$ and contact angles $\gamma_{i}$ 's. The inequalities (4) and (5) say that the wetted regions of a spanner in unbalanced polyhedral cone have bigger or equal area than the wetted region of the spherical spanner.

In [Park 2005], it was shown that every ring type spanner in a wedge is actually spherical. In this context, we ask two questions:

- Is every spanner in a wedge or in a triangular pyramid spherical?

- Is every spanner in an unbalanced polyhedral cone in $\mathbb{E}^{n+1}$ spherical?

\section{References}

[Concus et al. 2001] P. Concus, R. Finn, and J. McCuan, "Liquid bridges, edge blobs, and Scherktype capillary surfaces", Indiana Univ. Math. J. 50:1 (2001), 411-441. MR 2002g:76023 Zbl 0996. 76014

[McCuan 1997] J. McCuan, "Symmetry via spherical reflection and spanning drops in a wedge", Pacific J. Math. 180:2 (1997), 291-323. MR 98m:53013 Zbl 0885.53009

[Park 2005] S.-h. Park, "Every ring type spanner in a wedge is spherical”, Math. Ann. 332:3 (2005), 475-482. MR 2006h:53008 Zbl 02190811

Received March 7, 2007. Revised July 28, 2007.

\section{SUNG-HO PARK}

Korea Institute for AdVANCEd Study HoEgiro 87

207-43 CHEONGNYANGNi 2-DONG, DONGDAEMUN-GU

SEOUL 130-722

Korea

shubuti@kias.re.kr 